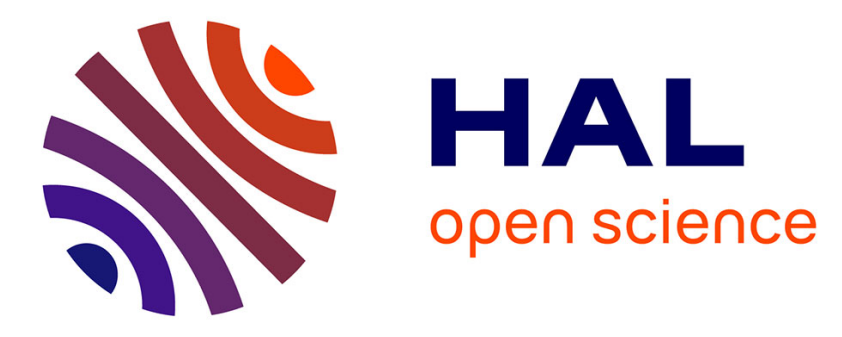

\title{
Organisation et évolution de la science au Brésil
}

Jean-Pierre Briot

\section{To cite this version:}

Jean-Pierre Briot. Organisation et évolution de la science au Brésil. Histoire de la recherche contemporaine: la revue du Comité pour l'histoire du CNRS , 2018, Histoire et structure de la coopération scientifique entre le Brésil et la France, VII (2), pp.127-137. 10.4000/hrc.2337 . hal-01840936

\section{HAL Id: hal-01840936 https: / hal.sorbonne-universite.fr/hal-01840936}

Submitted on 10 Aug 2019

HAL is a multi-disciplinary open access archive for the deposit and dissemination of scientific research documents, whether they are published or not. The documents may come from teaching and research institutions in France or abroad, or from public or private research centers.
L'archive ouverte pluridisciplinaire HAL, est destinée au dépôt et à la diffusion de documents scientifiques de niveau recherche, publiés ou non, émanant des établissements d'enseignement et de recherche français ou étrangers, des laboratoires publics ou privés. 


\title{
Histoire de la recherche contemporaine
}

La revue du Comité pour l'histoire du CNRS

Tome VII N ${ }^{\circ} 2 \mid 2018$ :

Histoire et structure de la coopération scientifique entre le Brésil et la France Dossier: Histoire et structure de la coopération entre le Brésil et la France

\section{Organisation et évolution de la science au Brésil}

\author{
JEAN-PIERRE BRIOT
}

p. $127-137$

\section{Texte intégral}

Un territoire immense, des ressources naturelles abondantes, un tiers de la biodiversité de la planète... le Brésil est un géant, confronté à de nombreux défis économiques, politiques et sociaux, mais qui a su développer une recherche scientifique de niveau mondial.

Il est de coutume de dire que le Brésil est un géant. C'est le 5 ème État le plus grand au monde par la superficie (8,5 millions de km2, environ 16 fois la taille de la France), juste derrière les États-Unis. Il couvre près de la moitié de la superficie de l'Amérique du Sud et est le plus grand État de l'hémisphère sud. Notons que la plus longue frontière de la France avec un autre pays est avec le Brésil $(730 \mathrm{~km})$. Le Brésil est une république fédérative, il compte 26 États et le district fédéral constitué depuis 1960 autour de Brasília, qui possède presque tous les attributs d'un État.

\section{Les grandes caractéristiques du Brésil}

\section{Ressources}

Les ressources naturelles du Brésil sont exceptionnelles : le pays est très riche en minerais et est par exemple le 1er producteur de minerai de fer. En 2006, d'immenses gisements de pétrole sous-marin ont été découverts près de la côte du sud-est (le pré- 
$\mathrm{sal}^{1}$ ). Il faut par ailleurs souligner l'excellente matrice énergétique du pays, avec une majorité de renouvelable : $45 \%$ (au 1er rang mondial), dont une majorité d'hydroélectrique (90 \% de l'électricité) et avec une avance en matière de biocarburants. Le Brésil est également un pays majeur en termes de biodiversité : il regroupe un tiers de la biodiversité mondiale et possède environ un cinquième des réserves d'eau douce.

3 Le Brésil a une population d'environ 213 millions d'habitants (5ème place mondiale ${ }^{2}$ ), là encore très riche en diversité. La densité est d'environ 25 habitants par km2, et la population est extrêmement urbanisée, même en Amazonie. Le développement du pays s'est fait à partir de la côte Atlantique, où se situe encore la majorité de la population, notamment dans la région Sud-est (autour de Rio de Janeiro et São Paulo). La création ex-nihilo à la fin des années 1950 de la nouvelle capitale fédérale Brasília voulait symboliser la conquête de l'intérieur du pays et le développement de villes nouvelles.

\section{Économie et commerce}

L'économie du pays a fortement progressé 3 entre 2005 et 2015 et le Brésil est actuellement la gème puissance économique mondiale, juste après l'Italie, avec un PIB en 2017 d'environ 2,1 milliards de dollars. Il était au 7ème rang en 2013 et a depuis rétrogradé. Le pays est en effet plongé depuis 2015 dans un relatif marasme économique. Le Brésil, il est vrai, a depuis une décennie eu trop tendance à se reposer sur la richesse de ses matières premières (minerais, soja, viande...) et sur un marché intérieur relativement protégé, avec par exemple un tiers des mesures protectionnistes adoptées en 2012 dans le monde. Les exportations sont ainsi dominées par les matières premières (minerais et agroalimentaire). Le pays compte cependant un certain de nombre de succès en termes d'innovation technologique, dans les domaines suivants :

- L'agro-alimentaire, où il a su investir dans l'innovation à travers l'institution publique fleuron EMBRAPA (équivalent de l'INRA) avec de grands succès à l'exportation ;

- L'énergie, avec l'extraction du pétrole en eau profonde, dans lequel le Brésil est leader, tiré par son entreprise pilote Petrobras, ainsi que dans les biocarburants, dans lequel le pays a acquis une avance technologique et pratique ;

- L'aéronautique, avec son pôle autour de l'entreprise Embraer et ses succès à l'exportation.

\section{Défis}

5 Le grand défi économique est la transition d'une économie fondée à la fois sur un vaste marché intérieur en partie protégé et sur l'exportation de matières premières, vers une économie basée sur la connaissance et l'innovation. L'innovation est effectivement assez faible au Brésil, avec trop peu de brevets, notamment par rapport à sa production scientifique devenue de bon niveau.

$\mathrm{Au}$ niveau socio-économique, le pays reste profondément inégalitaire et connait de sérieux problèmes de sécurité (un taux d'environ 30 homicides pour 100000 habitants). Un autre problème concerne le manque d'infrastructures, dans un pays il est vrai immense et au développement très hétérogène. Un défi est en conséquence de rééquilibrer en partie les régions traditionnellement peu développées, telles le Nord-est et le Nord, par rapport aux régions les plus riches, en premier lieu le Sud-est.

Un autre grand défi est le système politique, clientéliste et corrompu. Deux récents scandales de surfacturation et détournement de fonds publics, ceux du " Mensalão »4 puis celui actuel du «Petrolão » (en référence à Petrobras), illustrent la nature et l'échelle des problèmes. Pour la première fois depuis longtemps, la justice a pris la mesure du problème avec l'opération " Lava Jato »5 et de nombreux responsables politiques et d'entreprises de premier plan se sont retrouvés en prison ferme, tels l'ancien président Lula ou encore Marcelo Oderbrecht, le patron de la première 
entreprise du bâtiment. La conjonction de ces scandales répétés, de l'inflation et d'investissements pharaoniques pour la Coupe du Monde puis les Jeux Olympiques, alors que les services publics de base (transports, hôpitaux...) sont sous-financés et peu efficaces, a désenchanté les Brésiliens, même s'ils gardent une capacité remarquable d'adaptation et d'optimisme.

Mais le plus grand défi du pays reste l'éducation, surtout dans le primaire et le secondaire, l'enseignement supérieur, étant lui de bon niveau. Le système éducatif public de base reste encore inefficace et inégalitaire (seulement $14 \%$ des jeunes de 18 à 24 ans vont à l'université et les classes moyennes envoient leurs enfants dans des écoles privées).

\section{La science brésilienne}

La science et la technologie bénéficient depuis plusieurs années d'investissements publics croissants, au niveau fédéral et au niveau des États fédérés, même si depuis 2016, avec la crise économique et le déficit public, les budgets ont été significativement restreints. Au niveau géo-scientifique, en plus des relations avec les pays occidentaux, le Brésil aspire aussi à jouer un rôle important dans les coopérations "Sud-Sud ", avec l'Amérique latine mais aussi l'Afrique.

\section{Statut mondial}

On peut observer depuis une vingtaine d'année la part croissante du Brésil dans l'activité scientifique internationale à travers différents critères (production scientifique, financement, nombre d'étudiants, proportion de professeurs d'universités ayant des doctorats...). Le Brésil se situe ainsi actuellement au 15ème rang mondial pour la production scientifique (publications), avec un pourcentage d'environ 2,5\%. Ce qui est le plus remarquable est sa récente forte progression, la 2ème plus grande progression (après la Chine) entre 2000 et 2010 : une multiplication par 8 des articles dans des revues scientifiques en 18 ans (Figure 1).

Figure 1. Évolution en pourcentage entre 2002 et 2012 des 15 pays les plus productifs

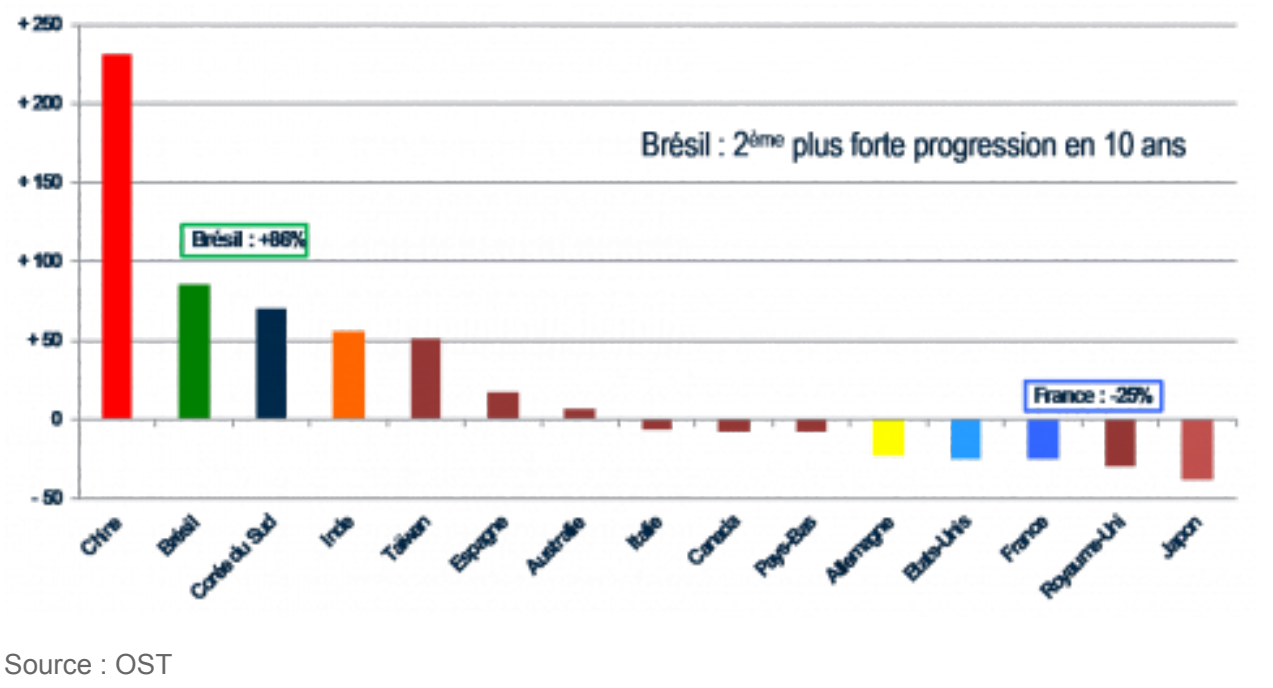

Une des faiblesses est l'impact, relativement faible par rapport à la production scientifique. Mais comparativement à la Chine, le rapport évolution entre citation et production reste plus raisonnable (Figure 2). D'autres faiblesses du Brésil sont le faible nombre de brevets, la trop faible internationalisation et enfin la bureaucratie encore très lourde.

Figure 2. Évolution comparée de la production scientifique et de l'impact de la Chine et du Brésil 


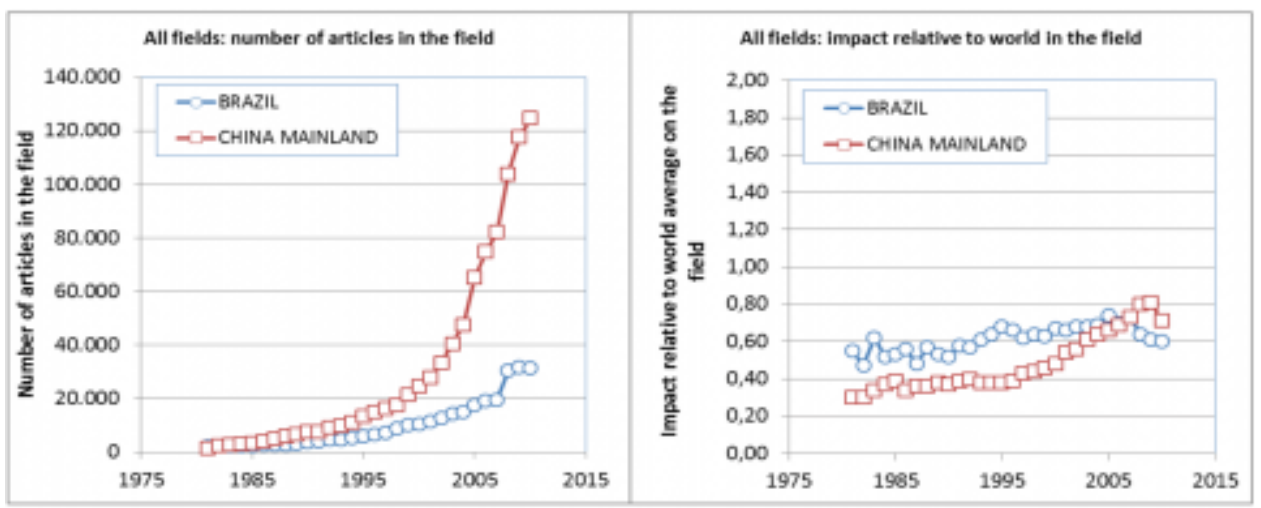

Source : FAPESP

12 Une analyse des coopérations du Brésil avec ses voisins d'Amérique du Sud (Figure 3) montre la grande activité de collaboration à l'intérieur de l'Amérique du Sud, le rôle leader du Brésil, mais également son profil encore un peu trop autarcique avec une proportion assez importante des publications sans collaboration internationale, surtout si l'on compare avec des pays tels que l'Argentine et le Chili.

Figure 3. Analyse comparée des collaborations scientifiques des pays de l'Amérique du Sud 


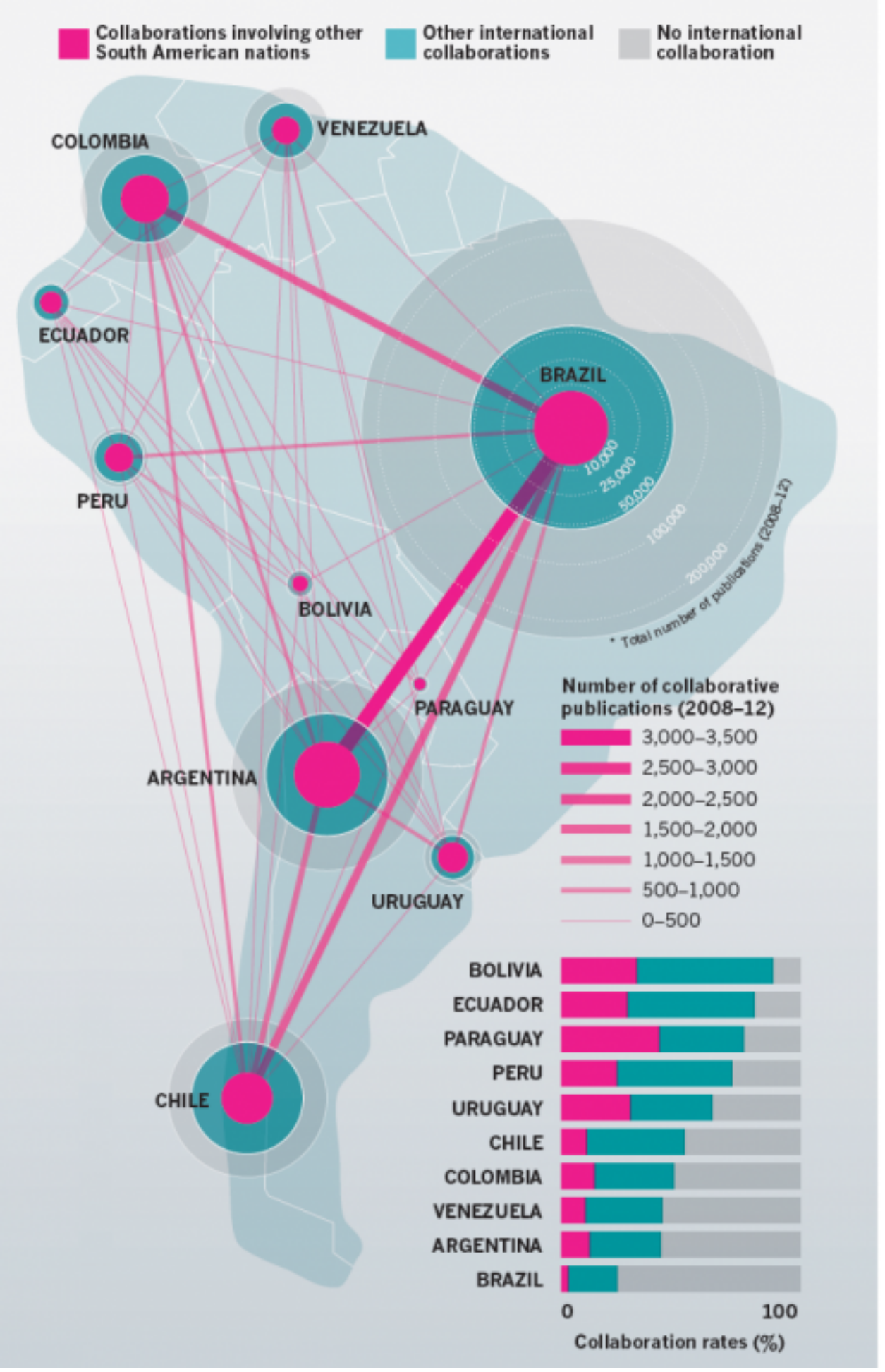

Source : Nature, vol. 510, № 7504, pp 187-306, 12 juin 2014

13 Si l'on analyse les domaines scientifiques (Figure 4), on voit l'excellence du pays en matière de biologie et écologie appliquées, du fait de ses caractéristiques propres (Amazonie, maladies tropicales) et de son implication dans l'agro-alimentaire.

\section{Figure 4. Indice de spécialisation du Brésil}




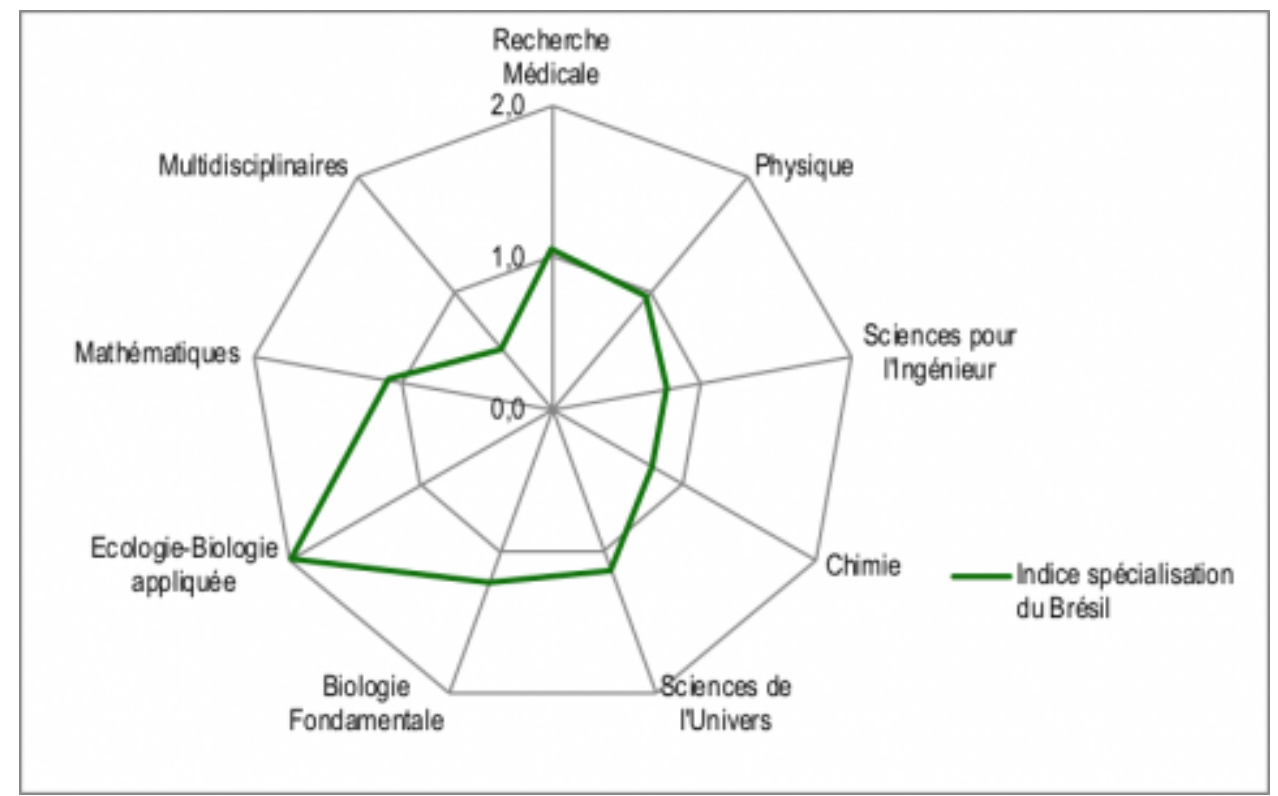

Source : Science Citation Index (Thomson-Reuters), Traitement : CNRS-SAP2S

\section{Ressources : budget et chercheurs}

Les investissements dans la recherche ont légèrement progressé depuis une quinzaine d'années. En 2006, ils représentaient environ $1 \%$ du PIB, en 2010, 1,24 \% et actuellement environ 1,3 \% (Figure 5). La valeur actuelle laisse cependant le Brésil pour le moment encore distant de la moyenne des pays occidentaux (de l'ordre de $2 \% \mathrm{du}$ PIB) ou de locomotives telles que la Corée du sud (4\% du PIB). Il faut également souligner que la majorité des investissements en R\&D au Brésil viennent du public, à l’inverse par exemple de la Corée du sud. Au Brésil, ils se répartissent de la façon suivante : 34 \% gouvernement fédéral, 18 \% États, $48 \%$ entreprises (publiques et privées).

Figure 5. Comparaison de la part du PIB affectée à la recherche

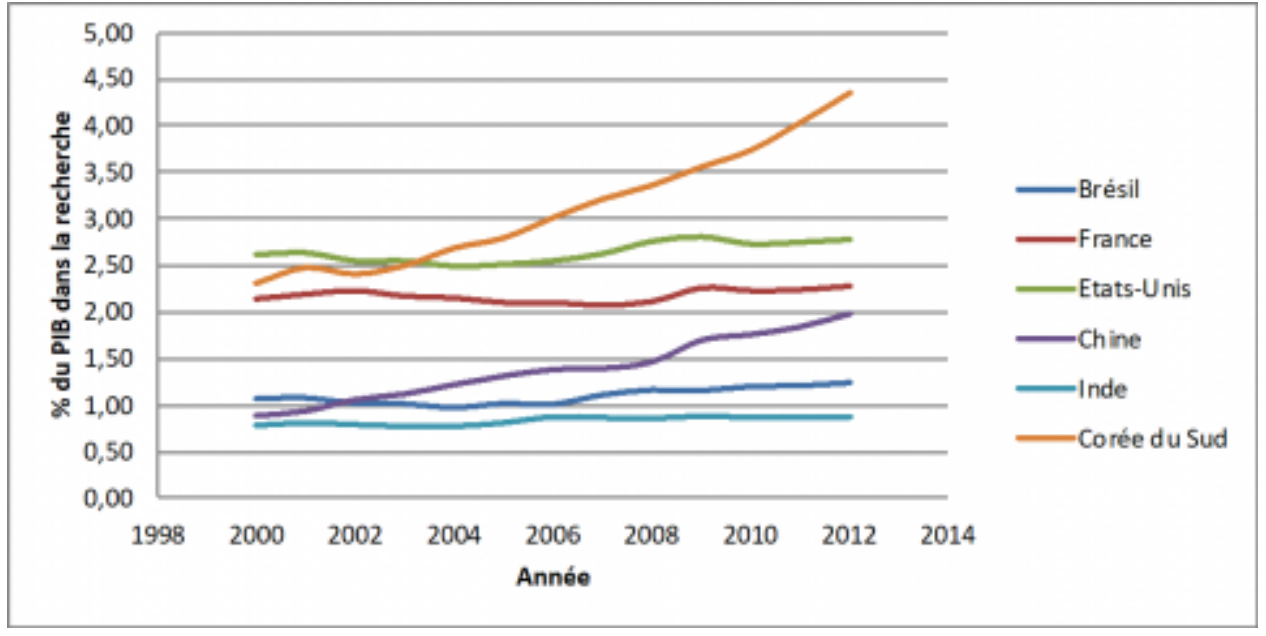

Source : MCTIC

Le Brésil compte environ 266 ooo chercheurs. Si l'on considère maintenant l'ensemble du personnel de recherche (chercheurs et appui technique et administratif), cela représente le double, soit un ratio de 2,6 pour 1 ooo habitants, environ un cinquième du ratio français. La plupart des chercheurs au Brésil ont un statut d'enseignant-chercheur des universités. Les chercheurs à plein temps sont peu nombreux et presque exclusivement affectés aux instituts de recherche publics. La répartition des chercheurs en fonction de leur institution de rattachement ${ }^{6}$ est : $95 \%$ dans l'enseignement supérieur, 6,1 \% dans les instituts gouvernementaux, 1,7 \% dans les entreprises (publiques ou privées) et 0,5\% dans le secteur associatif. 
La production de docteurs au Brésil a progressé de manière continue, de 1005 en 1987 jusqu'à 12217 en 2011, ce qui représente respectivement $3 \%$ et $25 \%$ de la production annuelle équivalente des États-Unis et montre ainsi le chemin accompli. Les universités fédérales ont majoritairement absorbé cette croissance de docteurs, pour la plupart devenus enseignants chercheurs (40 ooo docteurs enseignants chercheurs en 2000 et 115000 en 2012) (Figure 6). Notons que les diplômes en science ou en ingénierie restent faibles : de l'ordre de $11 \%$ de tous les diplômes en 2007, environ la moitié de la moyenne des pays de l'OCDE. Et même si le nombre d'ingénieurs formés a récemment grandi, il atteint actuellement un plateau (de l'ordre de 47000 diplômes en 2009) et ne couvre que la moitié de la demande intérieure. Une étude fort intéressante proposée par Naercio Menezes en 2015 (Figure 7) compare le Brésil et la Corée du sud en matière de corrélation entre l'évolution de la productivité et du nombre d'années d'études moyen. Elle tend à montrer que la Corée du sud, à l'inverse du Brésil, a profité à plein de l'allongement des études.

Figure 6. Secteur d'emploi des docteurs

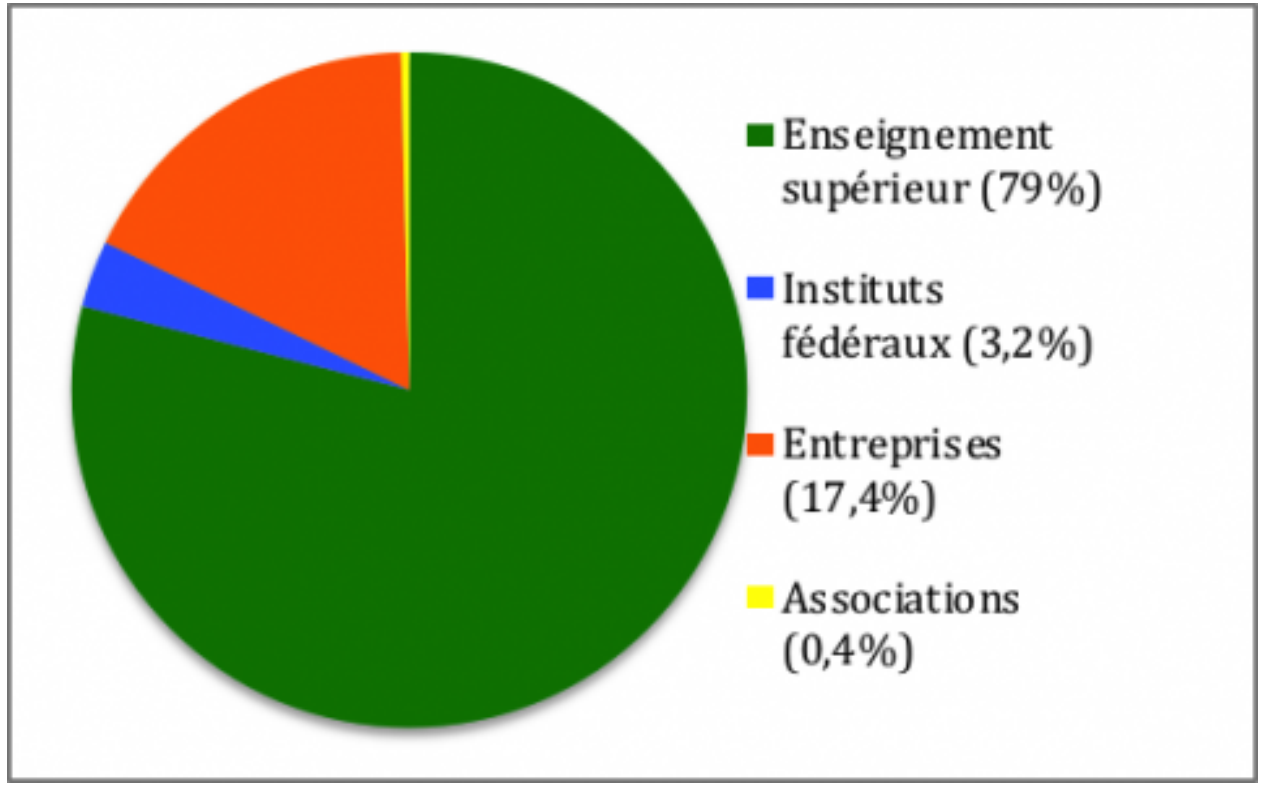

Source : MCTIC

Figure 7. Évolution comparée pour le Brésil et la Corée

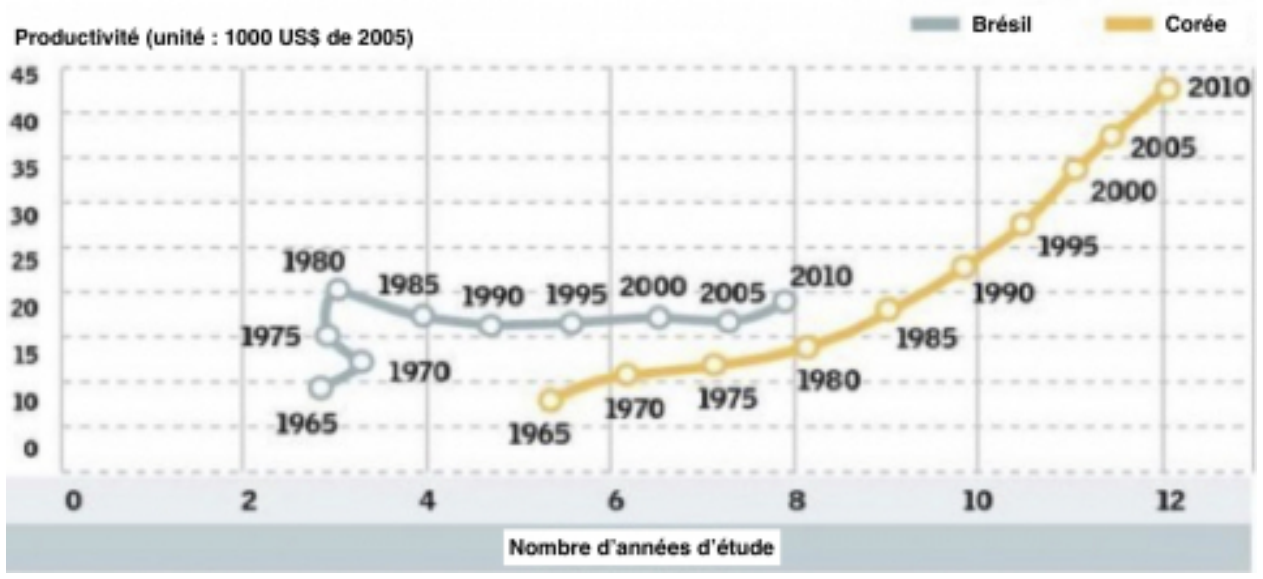

Comparaison de la productivité et du nombre moyen d'années d'étude

Source : Barro et Lee, 2010 ; OECD ; (Timmer et al., 2014), Élaboration : (Menezes, 2015).

\section{Organisation du système scientifique brésilien}

L'organisation de la recherche au Brésil (Figure 8) reflète la structure fédérale du pays, avec une indépendance (et une compétition, mais aussi une complémentarité) entre le niveau fédéral et le niveau des États fédérés. Le système scientifique brésilien 
offre par ailleurs une séparation claire entre les opérateurs de recherche (pour la majorité des universités, ainsi que des instituts de recherche fédéraux) et les agences de financement.

Figure 8. Organisation de la recherche au Brésil

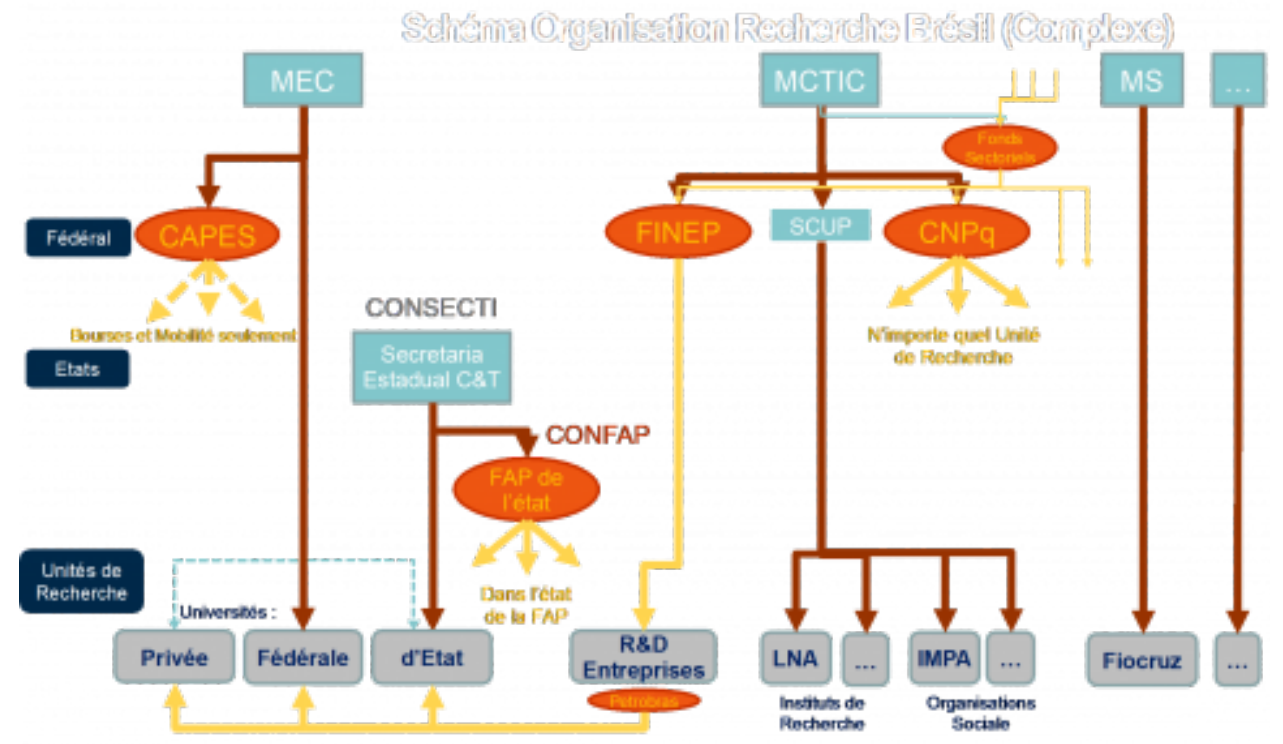

MEC : ministère de l'Éducation ; MCTIC : ministère de la Science, Technologie, Innovation et Communication ; MS : ministère de la Santé ; En orange : Agences de financement de la recherche ; En gris : Opérateurs de recherche

\section{Niveau fédéral}

Le niveau fédéral est pour ce qui est de son niveau de contrôle situé dans la capitale fédérale, Brasília. Les agences et instituts qui en dépendent peuvent être eux localisés soit à Brasília, soit pour la plupart des instituts de recherche dans différentes localités du pays, avec une concentration dans la région Sud-Est (dans les États de Rio de Janeiro et de São Paulo).

\section{Ministère de la science et institutions affiliées}

Le ministère de la Science, de la Technologie, Innovation et Communication (MCTIC) est l'acteur principal de la promotion de la recherche et de la technologie du pays. Il est responsable du Plan d'action de la science, de la technologie et de l'innovation pour le développement national, élaboré en coordination avec d'autres ministères (Éducation, Industrie, Santé...). Les deux principales agences de financement de la recherche dépendant de ce ministère sont le CNPq et la FINEP.

Le $\mathrm{CNPq}^{7}$, dont le siège est à Brasília, est la première agence de financement de la recherche scientifique au Brésil et le premier partenaire historique du CNRS (avec un accord signé en 1975). Il propose de nombreux types d'appels d'offre pour le financement de projets de recherche, pour des bourses, et pour des projets de coopération internationale. Le CNPq héberge également la plate-forme électronique de CVs scientifiques nommée LATTES ${ }^{8}$, sur laquelle les chercheurs tiennent à jour leur CV scientifique. Cette uniformisation de l'accès et du format s'avère très pratique et la plate-forme est utilisée comme base d'évaluation par différents organismes au Brésil. La FINEP (Financiadora de Estudos e Projetos), localisée à Rio de Janeiro, est quant à elle plus axée sur le financement de projets à visée technologique en partenariat avec les entreprises.

En ce qui concerne les opérateurs de recherche, le MCTIC possède également dans ses attributions ${ }^{9} 22$ unités de recherche scientifique et technologique. Ces unités de recherche fédérales se répartissent en deux types, selon une subdivision administrative interne subtile, entre instituts de recherche et $"$ organisations sociales ${ }^{10}$. Parmi ces 22 
unités de recherche, on compte par exemple l'IMPA (mathématiques) avec lequel le CNRS possède une UMI, le LNCC (informatique) ou le LNBio (biosciences) qui sont les partenaires du CNRS dans deux LIA. Ces Instituts de recherche possèdent des programmes de pós-graduação ${ }^{11}$ pour former des maîtres et des docteurs. Ainsi, bien que n'étant pas des universités, ils possèdent certaines des caractéristiques d'universités " post-graduate » et donc ne dépendent pas des universités pour former leurs doctorants.

\section{Ministère de l'éducation et institutions affiliées}

Le ministère de l'Éducation (MEC) a également un rôle important dans l'activité scientifique au Brésil. La CAPES (Coordinação de Aperfeiçoamento de Pessoal de Nível Superior) est son agence dédiée au financement et à l'évaluation de l'enseignement supérieur. Cette agence évalue les programmes de pós-graduação des différents départements d'université, où se trouve la grande majorité des cadres de la recherche brésilienne. En outre, de manière analogue au CNPq, elle propose différents types de bourses pour étudiants, post-docs et professeurs invités, ainsi que des programmes de coopération internationale, dont le programme d'échanges franco-brésilien CAPESCOFECUB qui atteindra en 2019 ses 40 ans d'existence. Il a de manière cumulée financé plus de 800 projets et produit plus de 3000 docteurs (dont $20 \%$ de Français). La CAPES considère ce programme comme une référence et un modèle pour la coopération internationale scientifique et de formation des chercheurs.

Les opérateurs de recherche dépendant du MEC sont les universités fédérales. Il y en a une soixantaine, réparties dans l'ensemble du pays, avec une certaine concentration dans la région Sud-est, notamment dans l'État du Minas Gerais. Ce sont des universités de bon niveau voire parmi les meilleures du pays pour l'UFRJ (Rio de Janeiro) et l'UFMG (Minas Gerais), toutes deux situées entre le 30oème et le 400ème rang du classement de Shanghai.

\section{Autres ministères et institutions affiliées}

Certains autres ministères ont un poids non négligeable en matière scientifique. C'est notamment le cas du ministère de la Santé, qui dispose avec la Fundação Oswaldo Cruz (Fiocruz) d'un organisme de recherche en pointe dans le secteur biomédical, avec lequel le CNRS, ainsi que l'INSERM et l'Institut Pasteur, ont une collaboration forte. Un autre exemple est l'EMBRAPA (Empresa Brasileira de Pesquisa Agropecuária), analogue brésilien de l'INRA français, qui dépend du ministère de l'Agriculture. Ces instituts de recherche fédéraux, sont au même niveau que les Instituts de recherche fédéraux dépendant du MCTIC (bien que dépendant d'autres ministères) qui comme eux possèdent des programmes de post doctorat. Il existe enfin d'autres institutions fédérales dépendant du MCTI ou d'autres ministères, telles l'agence spatiale, la commission d'énergie nucléaire, que nous ne développerons pas ici.

\section{Niveau des États}

Chaque gouvernement d'État dispose d'un secrétariat à la Science et à la Technologie. La plupart des États prévoient dans leur propre constitution la redistribution d'un pourcentage des recettes budgétaires à destination de la recherche scientifique et technologique. Ces ressources sont gérées par des fondations agences de financement de la recherche appelées FAP (Fundação de Amparo a Pesquisa). Les États les plus riches ont les FAPs les plus puissantes (en premier lieu la FAPESP pour l'État de São Paulo). De manière analogue au CNPq et à la CAPES, les FAPs proposent des appels d'offre pour l'attribution de bourses, de soutien à la recherche, aux échanges scientifiques et à la diffusion de la science et de la technologie. 
Chaque État a son propre réseau d'universités d'État distinct des universités fédérales (par exemple dans la ville de Rio de Janeiro, l'UERJ (Universidade Estadual do Rio de Janeiro) dépend de l'État tandis que l'UFRJ (Universidade Federal do Rio de Janeiro) est fédérale. Les universités les plus prestigieuses restent les universités fédérales, sauf pour l'État de São Paulo, pour lequel la hiérarchie est inversée, les deux meilleures universités de l'État de São Paulo, et également du pays, étant la USP (Universidade de São Paulo) et l'UNICAMP (Universidade Estadual de Campinas). Ceci est dû à la place toute particulière de l'État de São Paulo, poumon économique du Brésil, qui apporte depuis longtemps un financement très important aux universités de l'État ainsi qu'à la FAPESP, la FAP de l'État de São Paulo. Un État riche tel que l'État de São Paulo garde une autonomie et une puissance qui lui permettent de rivaliser avec le niveau fédéral, par exemple pour ses programmes à l'international. Il existe ainsi un programme bilatéral de coopération en enseignement supérieur et recherche USP-COFECUB (donc spécifique à l'USP), distinct du Programme CAPES-COFECUB, pour l'ensemble des autres universités brésiliennes. L'État de São Paulo a véritablement eu et garde encore un rôle pilote. D'autres États riches, tels que l'État du Minas Gerais, ont suivi ses traces sans encore le rejoindre.

\section{Compétition, complémentarité et coordination entre Fédération et États}

Les deux systèmes parallèles de financement de la recherche et d'universités sont à la fois en compétition et complémentaires. Il existe des structures et des opérations de coordination au niveau des États à travers le CONSECTI, conseil qui fédère les secrétariats d'État à la Science et à la Technologie des États, et surtout à travers le CONFAP, conseil qui fédère les FAPs et a récemment pris beaucoup d'importance dans la coordination des FAPs. Au niveau national, il existe également des accords entre les agences fédérales (CNPq et FINEP) et les agences des États (FAPs), pour des cofinancements de programmes, tels par exemple le financement des INCTs (Instituto Nacional de Ciência e Tecnologia), programme du CNPq de réseaux thématiques d'équipes brésiliennes.

Outre les ministères mentionnés et les États, on compte deux autres sources majeures de financement de la recherche : 1) les fonds sectoriels (ex. : aéronautique, agronomie, Amazonie, pétrole...) dont les recettes proviennent notamment de contributions versées sur l'exploitation des ressources naturelles et d'impôts appliqués sur les produits industriels de certains secteurs, et qui alimentent des programmes spécifiques ainsi que le CNPq et la FINEP ; 2) Petrobras, la puissante ${ }^{12}$ entreprise publique du pétrole. Outre son centre de recherche propre, le CENPES, situé juste à côté du campus de l'UFRJ à Rio de Janeiro, Petrobras est un acteur majeur du soutien à la recherche via des financements et projets communs avec des laboratoires d'universités.

\section{Universités et institutions d'enseignement supérieur et de recherche}

Le Brésil est le plus grand système d'enseignement supérieur d'Amérique latine, avec 2013 institutions, en énorme majorité des universités. 88,1\% sont privées, mais la majorité de ces universités privées sont dédiées à la formation, notamment continue. Il existe cependant quelques universités privées d'un excellent niveau scientifique, notamment le réseau des universités Jésuites PUC (Pontifícia Universidade Católica), dont celle de Rio (la PUC-Rio), qui fait partie des meilleures universités brésiliennes.

Les institutions d'enseignement supérieur et de recherche offrent actuellement environ 3700 programmes de pós-graduação délivrant environ 10 ooo doctorats et 20000 masters par an. Le nombre d'étudiants dans l'enseignement supérieur au Brésil (qui a doublé entre 2002 et 2012) se monte actuellement à environ 7,5 millions. Pour 
mémoire, il y en a environ 2,4 millions en France, la proportion par rapport à la population totale étant sensiblement la même dans les deux pays.

\section{Réseaux de recherche}

Au cours de l'année 2008, le CNPq a lancé un nouveau dispositif de soutien aux projets de recherche collaboratifs : les Instituts Nationaux de Science et de Technologie (Institutos Nacionais de Ciência e TecnologiaI, INCT). Ils représentent des réseaux d'équipes sur une thématique stratégique pour le Brésil ou sur des frontières de la connaissance, visant à les mettre en réseau et à décloisonner les universités. Il y a actuellement 125 INCTs. Les projets sont financés pour une durée de six ans, renouvelable, et jusqu'à un montant de 3,4 millions $€$ chacun.

\section{Évaluation}

Le système d'évaluation scientifique brésilien repose principalement sur l'évaluation des programmes de pós-graduação des institutions d'enseignement supérieur et de recherche. La CAPES en est responsable et organise le système d'évaluation triennale des programmes depuis 1976. Il est intéressant de noter que l'organisation disciplinaire de l'évaluation inclut plusieurs catégories multidisciplinaires ainsi qu'une catégorie interdisciplinaire, avec pour chacune un comité spécifique (Briot, 2013). Cette spécificité du traitement de l'interdisciplinarité, qui nous paraît assez en avance sur certains plans, a un effet d'entraînement sur les politiques des autres acteurs, universités et agences de financement. Il est vrai que l'échelle des défis du pays (environnement, inclusion sociale...) rend probablement plus naturelle, et nécessaire, une approche interdisciplinaire.

La note d'évaluation d'un programme de pós-graduação par la CAPES a un impact sur l'ensemble des autres évaluations de la recherche : quand un chercheur soumet une proposition au programme CAPES-COFECUB, ou un étudiant à une bourse CAPES ou $\mathrm{CNPq}$, ou un chercheur étranger à une bourse d'accueil au Brésil, l'évaluation du dossier par la CAPES, ou par le CNPq, incorpore toujours et avec un poids important la note du programme de pós-graduação d'accueil.

La deuxième évaluation, également consultée lors de la plupart des soumissions, repose sur le CV du chercheur et notamment sa catégorie de bourse de productivité du $\mathrm{CNPq}^{13}$, disponible sur la plate-forme LATTES du CNPq contenant environ 2,5 millions de CVs. Beaucoup de chercheurs ne sont pas sélectionnés aux bourses de productivité, ou ne candidatent pas, auquel cas, il n'y a pas d'évaluation affichée des chercheurs.

On peut noter qu'il n'y a pas actuellement au Brésil de système d'évaluation unifié (global) des équipes ou laboratoires de recherche. La gouvernance brésilienne a d'ailleurs commencé à s'intéresser à l'expérience française en la matière (évaluations par l'AERES et par le Comité National du CNRS). Ainsi, en décembre 2013, a eu lieu une première expérience d'évaluation conjointe, par un comité mixte comprenant des évaluateurs externes, par l'IRD et le CNPq des deux LMI (Laboratoires Mixtes Internationaux) de l'IRD avec le Brésil.

\section{Innovation}

Le pays connaît un retard dans le domaine de l'innovation, son économie et son commerce extérieur sont trop fondés sur les matières premières et trop peu sur la technologie. En effet, en regard de son 15ème rang mondial pour la production scientifique, le Brésil se situe vers le 50ème rang en matière d'innovation et le nombre de brevets reste très bas (environ 600 et environ $0,1 \%$ des dépôts mondiaux). De plus la majorité des brevets est concentrée dans trois institutions publiques ou para-publiques : Petrobras, EMBRAPA et Fiocruz. Et même s'il y a un très grand nombre de PME et microentreprises (dont une partie liée au « secteur informel »), la plupart sont peu ou 
pas innovantes. L'activité en R\&D autour du pétrole et de l'énergie du géant Petrobras reste un des seuls domaines d'innovation performant au Brésil.

Le pourcentage des dépenses des entreprises en recherche, développement et innovation par rapport au PIB part en effet de très bas, il était de $0,85 \%$ en 2008, la moitié de la moyenne des pays de l'OCDE. Même s'il augmente très légèrement chaque année (3\% par an), le retard pris est trop important et l'accélération trop faible. De plus, la majorité de ce financement part souvent dans l'achat de machines et équipements, plus que dans la réalisation de projets de recherche et d'innovation.

Depuis quelques années, on sent une grande prise de conscience de la nécessité d'une culture de l'innovation et une volonté de passer progressivement à une économie de la connaissance. La Corée du sud est régulièrement citée en exemple. Une des actions en 2013 a été la création de l'EMBRAPII (Empresa Brasileira de Pesquisa e Inovação Industrial). Son objectif est de faciliter l'innovation dans les entreprises, via une interaction entre la demande des entreprises et l'offre des laboratoires, en s'inspirant en partie du modèle de l'Institut Fraunhofer allemand ${ }^{14}$. Le système est différent d'appels à projets de partenariat plus classiques, tel que ceux proposés par la FINEP, l'agence de financement fédérale dépendant du MCTI dédiée à l'innovation et aux partenariats avec des entreprises. L'EMBRAPII sélectionne sur appels d'offres en amont des groupes (appelées unités) de recherche (actuellement 42), instituts ou centres de recherche publics d'universités ou d'instituts technologiques. Ces unités peuvent ensuite négocier directement des contrats avec des entreprises et recevoir des financements. 91 brevets sont déjà issus des 90 projets EMBRAPII terminés.

Au niveau des universités et instituts de recherche, la création d'agences d'innovation des laboratoires de recherche a été rendue obligatoire par la loi de l'innovation entrée en vigueur en octobre 2005. Si toutes les institutions se sont dotées de structures de valorisation, celles-ci sont encore peu développées. La plus importante et la plus dynamique est l'agence INOVA, rattachée à l'UNICAMP, suivie de près par celle de l'USP. Enfin, il existe plusieurs parcs technologiques de poids, souvent connexes et associés à des universités (USP, UNICAMP, UFRJ...). Deux exemples d'écosystèmes de R\&D sont en aéronautique autour du constructeur Embraer à São José dos Campos dans l'État de São Paulo et en exploration du pétrole en eau profonde autour de Petrobras à Rio de Janeiro.

\section{Conclusion}

À l'échelle de l'Amérique latine, le Brésil est un leader en recherche avec la moitié des publications et $60 \%$ du budget. Au niveau international, le pays a récemment beaucoup progressé, le nombre de publications brésiliennes dans des revues indexées a plus que doublé en 10 ans pour atteindre 20000 par an, passant devant la Russie ou la Suède. Le nombre de doctorats décernés a également doublé. Le Brésil est très bien placé à l'échelle mondiale dans certains secteurs tels que l'agronomie, les biocarburants et les maladies tropicales. Cependant, le nombre de publications ayant un fort impact reste faible, de même que le nombre de brevets.

Le défi général pour le Brésil en recherche (et de manière plus générale) est de maîtriser d'un point de vue organisationnel la «transition de phase » consécutive à une croissance scientifique récente et rapide (passer de la quantité à la qualité, internationaliser, débureaucratiser...).

\section{Bibliographie}

Briot Jean-Pierre, «Le soutien à l'interdisciplinarité au Brésil - Les spécificités et l'impact du système d'évaluation des programmes de post-graduation », Hermès, CNRS-Editions, (67) :136137, novembre 2013. Numéro spécial : « Discipline, interdisciplinarité, indiscipline », coordonné par J.-M. Besnier et J. Perriault.

Théry Hervé, Le Brésil - Pays émergé, Paris, Armand Colin, 2014.

DOI : 10.3917/geoec.073.0101 


\section{Annexe}

\section{Glossaire}

CAPES : Coordinação de Aperfeiçoamento de Pessoal de Nível Superior. L'agence fédérale d'évaluation et de financement de l'enseignement supérieur et de la recherche.

CNPq : Conselho Nacional de Desenvolvimento Científico e Tecnológico. L'acronyme CNPq provient du nom original «Conselho Nacional de Pesquisa ». L'agence fédérale de financement de la recherche scientifique.

CAPES-COFECUB : C'est le plus ancien et un des principaux programmes de coopération scientifique entre le Brésil et la France.

COFECUB : Comité Français d'Évaluation de la Coopération Universitaire et Scientifique avec le Brésil. C'est le comité d'évaluation français créé à l'occasion de la création du programme CAPESCOFECUB.

CsF : Ciência sem Fronteiras. Le programme brésilien de coopération scientifique internationale entre 2012 et 2016.

EMBRAPA : Empresa Brasileira de Pesquisa Agropecuária. La grande institution de recherche fédérale en agronomie, correspondante de l'INRA français.

EMBRAPII : Empresa Brasileira de Pesquisa e Inovação Industrial. La récente institution de financement de projets coopératifs entre universités et instituts de recherche et l'industrie.

FAP : Fundação de Amparo a Pesquisa. La fondation de financement de la recherche d'un État du Brésil.

FAPESP : Fundação de Amparo à Pesquisa do Estado de São Paulo.

FINEP : Financiadora de Estudos e Projetos, Rio de Janeiro. L'agence publique de financement des coopérations en R\&D entre universités et industrie.

FIOCRUZ : Fundação Oswaldo Cruz. Un institut fédéral de recherche biomédicale localisé à Rio de Janeiro.

IMPA : Instituto Nacional de Matemática Pura e Aplicada. Institut national de mathématique pure et appliquée. Un institut fédéral de recherche en mathématiques localisé à Rio de Janeiro.

INCT : Instituto Nacional de Ciência e Tecnologia. Institut national de science et de technologie. C'est un programme du CNPq de réseaux de recherche thématiques brésiliens.

LATTES : La plateforme de CVs scientifiques gérée par le CNPq.

MCTIC : Ministério da Ciência, Tecnologia, Inovações e Comunicações : Le ministère brésilien de la science, technologie, innovation et (télé)communication.

MEC : Ministério da Educação. Le ministère brésilien de l'éducation.

Programa de pós-graduação : Le programme d'étude après la graduation (4 ans) et qui inclut le master ( 2 ans) et le doctorat ( 4 ans). Cela correspond en France à une école doctorale.

pré-sal. Une réserve de pétrole qui s'est formée avant la formation d'une couche de sel et située en profondeur (jusqu'à $7 \mathrm{kms}$ ) sous une couche d'eau, une couche de roche et une couche de sel.

PUC-Rio : Pontifícia Universidade Católica do Rio de Janeiro. Une université privée jésuite d'excellent niveau.

USP : Universidade de São Paulo. Université de l'État de São Paulo. La plus grande université du Brésil et en général considérée comme la meilleure.

\section{Notes}

1 Il s'agit de très grandes réserves de pétrole en eau profonde à la hauteur de Rio de Janeiro et de São Paulo. Le nom de « pré-sal » provient du fait qu'elles se sont constituées avant (et donc sous) la formation d'une couche de sel.

2 Les premiers pays étant dans l'ordre : Chine, Inde avec chacune plus d'un milliard d'habitants, puis États-Unis et Indonésie.

3 Pour certains, le Brésil n'est plus un pays émergent mais un pays émergé (Théry, 2014).

4 « Mensalão » veut dire grande mensualité, en référence aux mensualités versées aux parlementaires de la majorité du gouvernement Lula pour monnayer leur soutien.

5 Le nom, qui signifie lavage haute pression, vient du fait que l'enquête a débuté en identifiant une station-service d'essence utilisée pour le blanchissement d'argent sale.

6 Selon les statistiques du MCTIC, en considérant les seuls chercheurs possédant un doctorat. Si l'on considère cette fois tous les chercheurs répertoriés en tant que tels, indépendamment de leur niveau d'étude, on arrive alors à une proportion de 17,6 \% en entreprises. Le total est légèrement supérieur à 100 \% car un même chercheur peut parfois appartenir à plus d'une catégorie.

7 Son nom complet est « Conselho Nacional de Desenvolvimento Científico e Tecnológico » (Conseil National de Développement Scientifique et Technologique), l'acronyme CNPq provenant de son nom original « Conselho Nacional de Pesquisas ».

8 Elle a été nommée en hommage à César Lattes, physicien nucléaire Brésilien, célèbre pour sa participation dans les années 1950 aux recherches sur le pion, vecteur essentiel de la force de 
cohésion du noyau atomique.

9 Plus précisément, c'est le Sous-Secrétariat de Coordination des Unités de Recherche (SCUP), directement rattaché au Secrétariat Exécutif du Ministre, qui gère l'ensemble de ces unités de recherche.

10 Ces dernières, plus récentes, ont plus d'autonomie de gestion.

11 La pós-graduação, littéralement la post-graduation (4 ans), est l'analogue de l'École doctorale en France. Elle inclut les programmes de master ( 2 ans) et de doctorat ( 4 ans).

12 Surtout depuis la découverte d’importantes réserves de pétrole en eau profonde, le " pré-sal », même si elle a perdu beaucoup de sa superbe avec le scandale du Petrolão.

13 Il s'agit d'un analogue de la prime d'excellence scientifique du CNRS. Le montant est d'environ 1000 Réals par mois (environ $250 €$ ). Les niveaux les plus élevés offrent en plus un montant équivalent au laboratoire du chercheur. En contrepartie, le chercheur doit effectuer des évaluations (en tant que pair) de divers dossiers soumis au CNPq (appels à bourses ou à projets).

14 Ainsi que la réussite en R\&D en agroalimentaire grâce à l'EMBRAPA, d'où le choix du nom si proche.

\section{Table des illustrations}

\begin{tabular}{|c|c|c|}
\hline \multirow{5}{*}{ 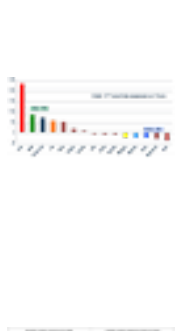 } & \multirow{4}{*}{$\begin{array}{c}\text { Titre } \\
\text { Crédits } \\
\text { UيLي } \\
\text { Fichier }\end{array}$} & $\begin{array}{l}\text { Figure 1. Évolution en pourcentage entre } 2002 \text { et } 2012 \text { des } 15 \text { pays les } \\
\text { plus productifs }\end{array}$ \\
\hline & & Source : OST \\
\hline & & http://journals.openedition.org/hrc/docannexe/image/2337/img-1.png \\
\hline & & image/png, 49k \\
\hline & Titre & $\begin{array}{l}\text { Figure } 2 \text {. Évolution comparée de la production scientifique et de l'impact } \\
\text { de la Chine et du Brésil }\end{array}$ \\
\hline & Crédits & Source : FAPESP \\
\hline & Uيسئي & http://journals.openedition.org/hrc/docannexe/image/2337/img-2.png \\
\hline & Fichier & image/png, 247k \\
\hline & Titre & $\begin{array}{l}\text { Figure 3. Analyse comparée des collaborations scientifiques des pays } \\
\text { de l'Amérique du Sud }\end{array}$ \\
\hline & Crédits & Source : Nature, vol. 510, № 7504, pp 187-306, 12 juin 2014 \\
\hline & Uي_ئي & http://journals.openedition.org/hrc/docannexe/image/2337/img-3.png \\
\hline & Fichier & image/png, $149 \mathrm{k}$ \\
\hline & Titre & Figure 4. Indice de spécialisation du Brésil \\
\hline & Crédits & $\begin{array}{l}\text { Source : Science Citation Index (Thomson-Reuters), Traitement : } \\
\text { CNRS-SAP2S }\end{array}$ \\
\hline & UيL & http://journals.openedition.org/hrc/docannexe/image/2337/img-4.png \\
\hline & Fichier & image/png, $61 \mathrm{k}$ \\
\hline & Titre & Figure 5. Comparaison de la part du PIB affectée à la recherche \\
\hline & Crédits & Source : MCTIC \\
\hline & URL & http://journals.openedition.org/hrc/docannexe/image/2337/img-5.png \\
\hline & Fichier & image/png, 24k \\
\hline & Titre & Figure 6. Secteur d'emploi des docteurs \\
\hline & Crédits & Source : MCTIC \\
\hline & Uي & http://journals.openedition.org/hrc/docannexe/image/2337/img-6.png \\
\hline & Fichier & image/png, 34k \\
\hline & Titre & Figure 7. Évolution comparée pour le Brésil et la Corée \\
\hline & Légende & Comparaison de la productivité et du nombre moyen d'années d'étude \\
\hline & Cré & $\begin{array}{l}\text { Source : Barro et Lee, } 2010 \text {; OECD ; (Timmer et al., 2014), } \\
\text { Élaboration : (Menezes, 2015). }\end{array}$ \\
\hline & URL & http://journals.openedition.org/hrc/docannexe/image/2337/img-7.jpg \\
\hline & Fichier & image/jpeg, 368k \\
\hline & Titre & Figure 8. Organisation de la recherche au Brésil \\
\hline & Crédits & $\begin{array}{l}\text { MEC : ministère de l'Éducation ; MCTIC : ministère de la Science, } \\
\text { Technologie, Innovation et Communication ; MS : ministère de la Santé ; } \\
\text { En orange : Agences de financement de la recherche ; En gris : } \\
\text { Opérateurs de recherche }\end{array}$ \\
\hline
\end{tabular}


UيLي http://journals.openedition.org/hrc/docannexe/image/2337/img-8.png

Fichier image/png, 139k

\section{Pour citer cet article}

Référence papier Jean-Pierre Briot, " Organisation et évolution de la science au Brésil », Histoire de la recherche contemporaine, Tome VII N² | 2018, 127-137.

Référence électronique

Jean-Pierre Briot, "Organisation et évolution de la science au Brésil », Histoire de la recherche contemporaine [En ligne], Tome VII N² | 2018, mis en ligne le 15 avril 2019, consulté le 10 août 2019. URL : http://journals.openedition.org/hrc/2337 ; DOI : 10.4000/hrc.2337

\section{Auteur}

\section{Jean-Pierre Briot}

directeur de recherche CNRS, membre du laboratoire d'informatique de Paris 6 (LIP6), a dirigé le bureau du CNRS à Rio de Janeiro, et est chercheur invité permanent à l'Université PUC-Rio (Pontifícia Universidade Católica do Rio de Janeiro), dans le laboratoire de génie logiciel.

Articles du même auteur

Introduction : histoire et structure de la coopération scientifique entre le Brésil et la

France [Texte intégral]

Paru dans Histoire de la recherche contemporaine, Tome VII N² | 2018

Entretien avec Luiz Davidovich, [Texte intégral]

Paru dans Histoire de la recherche contemporaine, Tome VII N² | 2018

\section{Droits d'auteur}

(C) Comité pour l'histoire du CNRS 